\title{
Análisis de la cultura tributaria de los comerciantes en zona de conflicto. Caso comerciantes ubicados en el centro de la ciudad de Ocaña, Norte de Santander*
}

Analysis of trader tax culture in a conflict zone. The case of traders in the city centre of Ocaña, Norte de Santander

Análise da cultura tributária em zona de conflito. Caso dos comerciantes localizados no centro da cidade de Ocaña, Norte de Santander

Naydu Judith Jácome Castilla ${ }^{\text {a }}$

Universidad Francisco de Paula Santander, Colombia

DOI: https://doi.org/10.11144/Javeriana.cc22.actc

njjacomec@ufpso.edu.co

ORCID: https://orcid.org/0000-0001-9241-0522

Recibido: 22/10/2019

Yasneidy Lorena Rizo Peñaranda

Aceptado: 09/07/2021

Universidad Francisco de Paula Santander, Colombia

Publicado: $31 / 12 / 2021$

ORCID: https://orcid.org/0000-0002-3563-2513

\section{Ilver Jaimes Carrascal}

Universidad Francisco de Paula Santander, Colombia

ORCID: https://orcid.org/0000-0002-7514-4709

\section{Resumen:}

Este artículo estudia la cultura tributaria de los comerciantes de la zona centro de la ciudad de Ocaña, Norte de Santander, con respecto al pago de los impuestos de industria y comercio y predial unificado. Es importante analizar cómo el recaudo de los impuestos afecta la generación de ingresos y el desarrollo económico en esta zona que hace parte de la región del Catatumbo marginada por la violencia y la ilegalidad. En términos metodológicos, se hizo una categorización de las actividades económicas y se determinó el nivel de cumplimiento mediante el conocimiento, la aceptación y el compromiso respecto a los tributos. Luego se aplicó una encuesta a la fuente primaria con el objetivo de analizar el nivel de cultura tributaria de los comerciantes. Como resultado de la investigación se evidencia que los contribuyentes de este sector poseen un nivel de cultura tributaria baja, dado que menos del $50 \%$ pagan de manera oportuna y consciente los impuestos municipales.

Código JEL: H20

Palabras clave: Cultura tributaria, impuesto predial, impuesto de industria y comercio, comerciantes.

\section{Abstract:}

This article discusses the tax culture of traders, specifically regarding payment of industry and trade taxes and unified property taxes in Ocaña's city centre. It is vitally important to analyse how tax collection affects income generation and economic development in the area, which forms part of the region of Catatumbo, marginalised by violence and illegality. In regard to the methodology, economic activities were categorized and the level of compliance was determined through knowledge of, acceptance of, and commitment to, taxes. A survey was applied to the primary source with the aim of analysing the level of tax culture of traders in the centre of Ocaña. This investigation has evidenced that taxpayers in this sector have a low level of tax culture, as less than 50\% consciously pay municipal taxes.

JEL Code: $\mathrm{H} 20$

Keywords: Tax culture, property tax, industry and trade tax, traders.

\section{Resumo:}

Este artigo estuda a cultura tributária dos comerciantes na área central da cidade de Ocaña, Norte de Santander, no que diz respeito ao pagamento de impostos sobre a indústria e o comércio e o predial unificado. É importante analisar como a arrecadação de impostos afeta em termos de geração de renda e desenvolvimento econômico nessa área que faz parte da região de Catatumbo marginalizada pela violência e ilegalidade. Em termos metodológicos, procedeu-se a uma categorização das atividades econômicas

Notas de autor

\footnotetext{
a Autora de correspondencia.E-mail: njjacomec@ufpso.edu.co
} 
e foi determinado o grau de cumprimento por meio do conhecimento, aceitação e comprometimento com os impostos. Em seguida, foi aplicada uma pesquisa à fonte primária para analisar o nível de cultura tributária dos comerciantes. Como resultado da pesquisa, fica evidente que os contribuintes desse setor têm um baixo nível de cultura tributária, uma vez que menos de $50 \%$ pagam conscientemente impostos municipais de forma oportuna.

Código JEL: H20

Palavras-chave: Cultura tributária, imposto imobiliário, indústria e imposto de comércio, comerciantes.

\section{Introducción}

Los impuestos son los aportes que hacen los contribuyentes al Estado, y representan la principal fuente de ingresos públicos con los cuales el gobierno financia sus gastos de funcionamiento y las inversiones. "Son deberes de la persona y del ciudadano: (...) contribuir al financiamiento de los gastos e inversiones del Estado dentro de conceptos de justicia y equidad" (Constitución Política, 1991, art. 95, num. 9). A su vez, el Estado debe retribuir a los ciudadanos en infraestructura, prestación de servicios y programas sociales que atiendan las necesidades de la sociedad.

Además de los impuestos nacionales, existen impuestos departamentales y municipales creados con el fin de que los entes territoriales puedan financiarse para promover el desarrollo local y departamental. Con relación a Ocaña, Norte de Santander, los impuestos municipales se encuentran estipulados en el Acuerdo No. 42 de 2009 donde se adopta el Estatuto de Rentas del municipio y se definen las normas legales, fundamentos y procedimientos administrativos y tributarios que regulan los ingresos de la jurisdicción (Concejo Municipal de Ocaña, 2009).

La Constitución Política de Colombia establece que toda persona desde que nace tiene derecho a ser ciudadano, hace parte de una ciudad y de un país y posee la responsabilidad de conocer y acatar las normas y leyes vigentes. Esto en la política fiscal implica que todo ciudadano deberá cumplir con el pago de sus obligaciones tributarias, teniendo en cuenta que estas surgen para contribuir con el financiamiento del Estado. Como consecuencia de esta obligatoriedad, se crea una relación entre el contribuyente y la Administración, donde el ciudadano de acuerdo con su capacidad económica, sufraga los gastos necesarios para el mantenimiento de las estructuras e instituciones del Estado (Economipedia, 2016).

De igual manera, teniendo en cuenta la importancia que tienen los impuestos para el desarrollo de un país es clave abordar la cultura tributaria, pues es la manera en que los contribuyentes manifiestan una conducta de cumplimiento de los deberes tributarios. Para ampliar el concepto es pertinente entender que la cultura según la Organización de las Naciones Unidas para la Educación, la Ciencia y la Cultura (UNESCO, 2017) consiste en los rasgos materiales y espirituales, intelectuales y afectivos de una sociedad; a través de ella discernimos los valores que definen el comportamiento moral y ético; y por ende determina la posición de las personas frente al cumplimiento de las obligaciones.

Un tema afín al pago de los impuestos es la cultura tributaria, entendida como la actitud del contribuyente que paga los impuestos de manera oportuna y bajo un marco de valores que como persona debe poseer. "Con la cultura tributaria se posibilita la aceptación voluntaria del pago por parte del contribuyente; razón por la que el buen uso de los recursos públicos constituye un elemento central de la cultura tributaria" (Bonilla, 2014, p. 23). En ese sentido, la importancia de la cultura tributaria radica en la aceptación de la persona en cuanto al deber constitucional de aportar al Estado y desarrollar un sentido de solidaridad para con los demás, por lo tanto, es indispensable que los contribuyentes entiendan la relación causal que existe entre el ciclo tributario y la distribución del gasto, para que sus comportamientos y acciones en torno de sus obligaciones con la tributación se orienten al cumplimiento voluntario y asuman las cargas fiscales de manera positiva.

En cuanto a la evasión tributaria, en Colombia esta alcanza niveles del $35 \%$, el porcentaje más alto de América Latina, pues según la Comisión Económica para América Latina y el Caribe (CEPAL) en Colombia el recaudo de impuestos respecto al Producto Interno Bruto (PIB) se encuentra en el 18,8 \% mientras el 
promedio de la región es de 22,8 \% (Portafolio, 2019). Además tiene las mayores tasas de evasión y elusión en el impuesto sobre la renta personal, y cerca al promedio en IVA y en renta empresarial (Concha, Ramírez, $\&$ Acosta, 2017). De esto se puede inferir que los colombianos no confían en la administración que hace el Estado con los recursos que reciben por el pago de impuestos y esto se debe a la corrupción en la que se encuentra inmerso el país (Departamento Administrativo de Hacienda y Secretaría de Educación Municipal de Cali, 2015).

De acuerdo con Abondano, Peña \& Parada (2018), casi la mitad de los ingresos municipales provienen de transferencias nacionales, mientras que la suma de los impuestos municipales (industria y comercio, predial unificado, entre otros) no representa ni la tercera parte de estos recursos. Entonces, la mayoría de los municipios del país se financian con los recursos que reciben de la nación a través del Sistema General de Participaciones - SGP (44,9\%), mientras que los recursos propios que generan por ingresos tributarios representan el 30,8\% (DNP, 2015). De manera análoga, hay 5 mil millones de pesos en cartera vencida por concepto de impuestos no pagados, según lo informó el Secretario de Hacienda de Ocaña, evidenciando una alta evasión tributaria (La Opinión, 2018), esto ocasiona que la ciudad no tenga los suficientes recursos para obras de bienestar social.

Teniendo en cuenta la importancia de los ingresos tributarios para los departamentos y municipios y el deber del cumplimiento de la obligación tributaria, la investigación tomó como población de estudio a los comerciantes que se encuentran ubicados en la zona centro de Ocaña, debido a que este sector es el punto de convergencia del comercio (Páez, 2014), donde se llevan a cabo las actividades económicas de mayor importancia para el desarrollo regional (Comercio Local, 2017).

Esta investigación tiene como objetivo primordial analizar la cultura tributaria de los comerciantes ubicados en la zona centro de la ciudad de Ocaña, teniendo en cuenta que hace parte de la región del Catatumbo (Centro Nacional de Memoria Histórica, 2017), la cual ha sido permeada por el flagelo de la industria del narcotráfico, provocando una desarticulación del tejido social afectando la gobernabilidad de la ciudad e incrementando las actividades ilegales. En consecuencia, se presenta una crisis en el sector agropecuario, bajos niveles de calidad de vida, altos índices de desempleo, cultura del dinero fácil y precarias condiciones de la infraestructura social (Cañizares, Cárdenas, \& Velasco, 2015).

Por consiguiente, se recurrió a una metodología descriptiva con la finalidad de analizar la cultura tributaria de los contribuyentes objeto de estudio, frente al pago de los impuestos de industria y comercio, avisos y tableros y predial unificado, recolectando la información a través de la aplicación de encuestas, con el propósito de establecer si existe y en qué grado cultura tributaria.

\section{Referencial teórico}

En la formulación y el desarrollo de la investigación, desde los planteamientos relacionados con la problemática presentada y el objeto de estudio, se utilizaron bases teóricas desde el contexto de la cultura tributaria, el nivel de cumplimiento de las obligaciones fiscales y los factores que influyen en la evasión de los impuestos de industria y comercio, su complementario avisos y tableros y el predial unificado; postulados que se emplearon como herramienta para la interpretación de los resultados, permitiendo analizar la cultura tributaria de los comerciantes en la zona centro de la ciudad de Ocaña, Norte de Santander.

\section{De la obligación a la cultura tributaria}

El sistema fiscal en Colombia y el conjunto de leyes contemplados en el régimen normativo del país constituyen un mecanismo de exigibilidad en el pago de impuestos por parte de los contribuyentes, para que el Estado obtenga los ingresos que garanticen la administración pública. 
La Constitución Política de Colombia establece que es deber de todo ciudadano cumplir las disposiciones legales en la presentación y pago de los impuestos, entendido esto, como la obligación sustancial establecida en el artículo 1 del Estatuto Tributario: "La obligación tributaria sustancial se origina al realizarse el presupuesto o los presupuestos previstos en la ley como generadores del impuesto y ella tiene por objeto el pago del tributo" (DIAN, 2020, art 1.). Siendo responsabilidad de todo ciudadano contribuir con la financiación del Estado realizando el pago de los impuestos a cargo, según la normatividad vigente.

En este orden de ideas, y partiendo de la premisa que el incumplimiento de las leyes genera sanciones para quien no cumpla con el ordenamiento legal, poder establecer el comportamiento de los contribuyentes en el pago de los impuestos, es de suma importancia para el Estado, las entidades reguladoras y recaudadores de los impuestos en Colombia.

Es preciso determinar que el comportamiento de los contribuyentes y su deber sustancial y formal de pagar impuesto está relacionado con el concepto de cultura tributaria, concebida como el conjunto de información, aunado al grado de conocimiento que poseen los ciudadanos sobre los impuestos, integrado al conjunto de percepciones, criterios, hábitos y actitudes que la sociedad tiene sobre la tributación (Villasmil, Fandiño, \& Alvarado, 2018).

Para la investigación fue necesario medir el grado de conocimiento tributario a través del entendimiento que poseen los comerciantes en cuanto a la obligación formal, sustancial y lo establecido en el Estatuto Municipal frente a los tributos, pues en la medida de que el contribuyente esté correctamente informado acerca de la legislación y de los beneficios que conlleva la oportuna declaración y pago de los impuestos, esto incidirá en el recaudo tributario y definirá su conducta en el cumplimiento de los deberes tributarios.

De igual forma, fue conveniente determinar la aceptación que poseen los comerciantes de cumplir las obligaciones tributarias, mediante la verificación de la consciencia tributaria, entendiéndose esta como la interiorización en los individuos de los deberes tributarios fijados por las leyes, para cumplirlos de una manera voluntaria, suponiendo que su cumplimiento acarreará un beneficio común para la sociedad (Colmenero, 2015).

Asimismo, en cuanto al compromiso que tienen los comerciantes frente al pago de los impuestos, se consideró la obligación formal de los contribuyentes, haciendo referencia al proceso, procedimiento o diligencia que se debe realizar ante el Estado para cumplir con el pago de los tributos (Gerencie, 2020), dado que especifica la forma y responsabilidad de contribuir con los gastos públicos.

En la tabla 1 se identifican los indicadores para medir el nivel de cultura tributaria. 
TABLA 1

Indicadores para medir el nivel de cultura tributaria

\begin{tabular}{|c|c|c|c|}
\hline $\begin{array}{c}\text { Cultura } \\
\text { tributaria }\end{array}$ & $\begin{array}{l}\text { Indicadores para } \\
\text { medir el nivel de } \\
\text { cultura tributaria }\end{array}$ & $\begin{array}{l}\text { Definición } \\
\text { conceptual }\end{array}$ & Reglamentación \\
\hline \multirow[b]{2}{*}{ Conocimiento } & $\begin{array}{l}\text { Obligación } \\
\text { tributaria sustancial }\end{array}$ & $\begin{array}{l}\text { Se origina al realizar los presupuestos } \\
\text { previstos en la ley como generadores } \\
\text { del impuesto y ella tiene por objeto el } \\
\text { pago del tributo. }\end{array}$ & $\begin{array}{l}\text { Estatuto Tributario. } \\
\text { Ley } 624 \text { de } 1989 \text {, art. } 1\end{array}$ \\
\hline & $\begin{array}{l}\text { Obligación } \\
\text { sustancial de los } \\
\text { impuestos } \\
\text { municipales de } \\
\text { Ocaña }\end{array}$ & $\begin{array}{l}\text { Es aquella que se origina al realizarse } \\
\text { el hecho generador del impuesto y } \\
\text { tiene por objeto el pago del tributo. }\end{array}$ & $\begin{array}{l}\text { Estatuto de Rentas del } \\
\text { municipio de Ocaña. } \\
\text { Acuerdo } 042 \text { de } 2009, \\
\text { art. } 5\end{array}$ \\
\hline \multirow{4}{*}{$\begin{array}{l}\text { Grado de } \\
\text { aceptación }\end{array}$} & Sujeto pasivo & $\begin{array}{l}\text { Es la persona natural o juríica, la } \\
\text { sociedad de hecho, la sucesión } \\
\text { iliquida o la entidad responsable del } \\
\text { cumplimiento de la obligación de } \\
\text { cancelar el impuesto, la tasa o la } \\
\text { contribución, bien sea en calidad de } \\
\text { contribuyente responsable o agente } \\
\text { retenedor. }\end{array}$ & $\begin{array}{l}\text { Estatuto de Rentas del } \\
\text { municipio de Ocaña. } \\
\text { Acuerdo } 042 \text { de } 2009, \\
\text { art. } 7\end{array}$ \\
\hline & Base gravable & $\begin{array}{l}\text { Es el valor monetario o unidad de } \\
\text { medida del hecho imponible, sobre el } \\
\text { cual se aplica la tarifa para } \\
\text { determinar el monto de la obligación. }\end{array}$ & $\begin{array}{l}\text { Estatuto de Rentas del } \\
\text { municipio de Ocaña. } \\
\text { Acuerdo } 042 \text { de } 2009, \\
\text { art. } 8\end{array}$ \\
\hline & Tarifa & $\begin{array}{l}\text { Es el valor determinado por la } \\
\text { normatividad, aplicable a la base } \\
\text { gravable y determinante de la renta, } \\
\text { contribución o participación. }\end{array}$ & $\begin{array}{l}\text { Estatuto de Rentas del } \\
\text { municipio de Ocaña. } \\
\text { Acuerdo } 042 \text { de } 2009, \\
\text { art. } 9\end{array}$ \\
\hline & $\begin{array}{l}\text { Tributos } \\
\text { municipales }\end{array}$ & $\begin{array}{l}\text { El Estatuto de Rentas de Ocaña } \\
\text { establece el pago de los impuestos: } \\
\text { predial unificado y de industria, } \\
\text { comercio y su complementario de } \\
\text { avisos y tableros. }\end{array}$ & $\begin{array}{l}\text { Estatuto de Rentas del } \\
\text { municipio de Ocaña. } \\
\text { Acuerdo } 042 \text { de } 2009, \\
\text { art. } 10\end{array}$ \\
\hline \multirow{2}{*}{ Compromiso } & Obligación formal & $\begin{array}{l}\text { Los responsables directos del pago } \\
\text { del tributo deberán cumplir los } \\
\text { deberes formales señalados en la ley } \\
\text { o en el reglamento, personalmente o } \\
\text { por medio de sus representantes, y a } \\
\text { falta de estos, por el administrador } \\
\text { del respectivo patrimonio. }\end{array}$ & $\begin{array}{l}\text { Estatuto Tributario. } \\
\text { Ley } 624 \text { de } 1989, \\
\text { art. } 571\end{array}$ \\
\hline & $\begin{array}{l}\text { Naturaleza del } \\
\text { impuesto predial }\end{array}$ & $\begin{array}{l}\text { Es un tributo de carácter municipal } \\
\text { que grava la propiedad inmueble } \\
\text { urbana y rural de la jurisdicción del } \\
\text { municipio, que se cobra sobre el } \\
\text { avalúo catastral o la declaración } \\
\text { privada de auto-avalúo. }\end{array}$ & $\begin{array}{l}\text { Estatuto de Rentas del } \\
\text { municipio de Ocaña. } \\
\text { Acuerdo } 042 \text { de } 2009, \\
\text { art. } 12\end{array}$ \\
\hline \multirow{5}{*}{ Compromiso } & $\begin{array}{l}\text { Hecho generador } \\
\text { del impuesto } \\
\text { predial }\end{array}$ & $\begin{array}{l}\text { Lo constituye la propiedad, posesión } \\
\text { o tenencia de un bien raíz urbano o } \\
\text { rural, en cabeza de una persona } \\
\text { natural o jurídica, incluidas las } \\
\text { personas de derecho público, en el } \\
\text { municipio de Ocaña. }\end{array}$ & $\begin{array}{l}\text { Estatuto de Rentas del } \\
\text { municipio de Ocaña. } \\
\text { Acuerdo } 042 \text { de } 2009, \\
\text { art. } 13\end{array}$ \\
\hline & $\begin{array}{l}\text { Sujeto pasivo del } \\
\text { impuesto predial }\end{array}$ & $\begin{array}{l}\text { Es la persona natural o juríicica, } \\
\text { pública o privada, propietaria o } \\
\text { poseedora del bien inmueble, los } \\
\text { administradores de patrimonios } \\
\text { autónomos por los bienes que de él } \\
\text { hagan parte, los herederos, } \\
\text { administradores o albaceas de } \\
\text { herencias yacentes o sucesiones } \\
\text { ilíquidas. Son solidariamente } \\
\text { responsables por el pago del } \\
\text { impuesto, el propietario y el poseedor } \\
\text { del predio. }\end{array}$ & $\begin{array}{l}\text { Estatuto de Rentas del } \\
\text { municipio de Ocaña. } \\
\text { Acuerdo } 042 \text { de } 2009, \\
\text { art. } 14\end{array}$ \\
\hline & $\begin{array}{l}\text { Hecho generador } \\
\text { del impuesto de } \\
\text { industria y } \\
\text { comercio }\end{array}$ & $\begin{array}{l}\text { Es la realización directa o indirecta } \\
\text { de cualauier actividad industrial, } \\
\text { comercial o de servicios en la } \\
\text { jurisdicción del Municipio de Ocaña, } \\
\text { ya sea que se cumplan de forma } \\
\text { permanente u ocasional, en inmueble } \\
\text { determinado, con o sin } \\
\text { establecimientos de comercio. }\end{array}$ & $\begin{array}{l}\text { Estatuto de Rentas del } \\
\text { municipio de Ocaña. } \\
\text { Acuerdo } 042 \text { de } 2009, \\
\text { art. } 33\end{array}$ \\
\hline & $\begin{array}{l}\text { Sujeto pasivo del } \\
\text { impuesto de } \\
\text { industria y } \\
\text { comercio }\end{array}$ & $\begin{array}{l}\text { Es la persona natural o jurídica o la } \\
\text { sociedad de hecho y demás entidades } \\
\text { de derecho público y privado, que } \\
\text { realicen el hecho generador de la } \\
\text { obligación tributaria, consistente en } \\
\text { el ejercicio de actividades } \\
\text { industriales, comerciales o de } \\
\text { servicios en la jurisdicción del } \\
\text { Municipio de Ocaña. }\end{array}$ & $\begin{array}{l}\text { Estatuto de Rentas del } \\
\text { municipio de Ocaña. } \\
\text { Acuerdo } 042 \text { de } 2009, \\
\text { art. } 32\end{array}$ \\
\hline & $\begin{array}{l}\text { Tarifas del } \\
\text { impuesto de } \\
\text { industria y } \\
\text { comercio }\end{array}$ & $\begin{array}{l}\text { Están establecidas en el estatuto de } \\
\text { renta municipal según la actividad } \\
\text { industrial, comercial y de servicios. }\end{array}$ & $\begin{array}{l}\text { Estatuto de Rentas del } \\
\text { municipio de Ocaña. } \\
\text { Acuerdo } 042 \text { de } 2009, \\
\text { arts. } 53 \text { al } 56\end{array}$ \\
\hline
\end{tabular}

Fuente: elaboración propia.

Para la determinación del nivel de cumplimiento en el pago de las obligaciones municipales, específicamente de los impuestos de industria y comercio, avisos y tablero y el predial unificado, se tuvo en cuenta la obligación tributaria formal y la obligación sustancial establecida en el Estatuto Tributario. Una de las características de la obligación tributaria sustancial es que nace de la ley cuando se realiza el hecho generador del impuesto y, por tanto, bajo un marco jurídico el sujeto activo, es decir, el municipio queda facultado para exigirle al contribuyente el pago de la obligación tributaria. Es así que la obligación 
sustancial crea en el sujeto pasivo la responsabilidad de tener conocimiento respecto al impuesto y de realizar el respectivo pago, es decir, ser consciente del compromiso adquirido y aceptar su obligación (Actualicese, 2017).

Respecto a la obligación formal de cumplir oportunamente la obligación del pago de impuestos es necesario realizar unos procedimientos y trámites, es decir, cumplir con disposiciones legales que la ley establece para que los contribuyentes cumplan sus obligaciones tributarias como declarar, expedir factura para quienes están obligados, inscribirse en el Registro Único Tributario (RUT), entre otros, demostrando así un compromiso por parte del comerciante para con el municipio.

Sobre los criterios para determinar el nivel de cumplimiento en el pago de los impuestos, se considera alto cuando el contribuyente cumple con la obligación sustancial y formal respecto a los impuestos de industria y comercio, avisos y tableros y predial unificado. El nivel es medio cuando se cumple con ambas obligaciones, es decir, acepta la obligación y realiza el pago, pero no tiene conocimiento respecto a los impuestos. Por último, cuando se realiza el pago del impuesto, pero no se tiene un conocimiento y no acepta la responsabilidad, es decir, no es consciente de la importancia de los impuestos como fuente de recursos para el municipio, se considera que el nivel de cumplimiento es bajo.

\section{Factores que influyen en el cumplimiento o evasión de los impuestos}

En los diferentes niveles de gobierno -nacional, departamental y municipal- se requieren el recaudo de impuestos y tributos con el fin de ejecutar los planes de desarrollo, de ahí la importancia de la concientización del deber de pagar impuestos por parte del contribuyente, para garantizar el cumplimiento de sus obligaciones y así evitar posibles sanciones. Por ello es importante conocer acerca de los factores que influyen en el pago, para que así el Estado establezca medidas gubernamentales que le permita un eficiente recaudo de los recursos públicos mitigando la evasión fiscal (Ramos \& Remigio, 2015).

Así pues, la percepción que se tenga sobre la tributación se manifiesta en el grado de aversión hacia los impuestos. Si los ciudadanos observan que los ingresos que el Estado obtiene se expresan en una mejor calidad de vida, la aversión hacia la tributación es menor (Bonilla, 2014). Por lo tanto, es indispensable generar consciencia y profundizar en la cultura tributaria de los contribuyentes, donde conozcan acerca de los impuestos, acepten la obligatoriedad y cumplan de manera oportuna y veraz.

Con relación a lo anterior, en la tabla 2 se detallan las causales de cumplimiento o evasión en los impuestos. Es importante resaltar que junto a estas causales se debe considerar el nivel de cumplimiento para identificar la cultura tributaria de los comerciantes. 
TABLA 2

Causas del cumplimiento o evasión en los impuestos

\begin{tabular}{|l|l|l|}
\hline $\begin{array}{c}\text { Causas de } \\
\text { cumplimiento o } \\
\text { evasión }\end{array}$ & \multicolumn{1}{|c|}{$\begin{array}{c}\text { Características de } \\
\text { cumplimiento o evasión }\end{array}$} & Normatividad que reglamenta \\
\hline Evitar sanciones & $\begin{array}{l}\text { Es un factor de cumplimiento, los } \\
\text { contribuyentes reconocen que el } \\
\text { incumplimiento de la obligación } \\
\text { sustancial de pagar impuestos } \\
\text { genera sanciones }\end{array}$ & $\begin{array}{l}\text { Régimen de sanciones del } \\
\text { impuesto predial e impuesto de } \\
\text { industria y comercio. Libro II del } \\
\text { Estatuto de rentas del Municipio } \\
\text { de Ocaña. Acuerdo 042 de 2009, } \\
\text { arts. 225-237. }\end{array}$ \\
\hline Beneficios sociales & $\begin{array}{l}\text { Este factor corresponde a los } \\
\text { beneficios a los cuales puede } \\
\text { acceder la comunidad. Los } \\
\text { contribuyentes esperan que el } \\
\text { pago de los impuestos sea para } \\
\text { financiar necesidades públicas, } \\
\text { como los gastos educativos, } \\
\text { sanitarios, en seguridad y } \\
\text { defensa, de infraestructuras, de } \\
\text { abastecimiento de agua potable, } \\
\text { de transporte público, entre otros, } \\
\text { los cuales promuevan al } \\
\text { desarrollo social y a la } \\
\text { satisfacción de las necesidades } \\
\text { sociales. }\end{array}$ & $\begin{array}{l}\text { Estatuto de Rentas del Municipio } \\
\text { de Ocaña. Acuerdo 042 de 2009. }\end{array}$ \\
\hline Desconocimiento & $\begin{array}{l}\text { Hace referencia a las exigencias } \\
\text { de la obligación formal tributaria, } \\
\text { para la presentación y pago de } \\
\text { impuestos. }\end{array}$ & $\begin{array}{l}\text { Acuerdo 0002 del 18 de } \\
\text { diciembre de 2018, donde se } \\
\text { establece calendario tributario, } \\
\text { plazos para los pagos del } \\
\text { impuesto predial e impuesto de } \\
\text { industria y comercio. }\end{array}$ \\
\hline Obligación & $\begin{array}{l}\text { Hace referencia a las exigencias } \\
\text { te la obligación sustancial } \\
\text { deben conos contribuyentes los deberes } \\
\text { fiscales en el desarrollo de sus } \\
\text { actividades económicas. }\end{array}$ & $\begin{array}{l}\text { Estatuto Tributario de Rentas del } \\
\text { Municipio de Ocaña. Acuerdo } \\
\text { 042 de 2009, arts. 5, 12 y 33. }\end{array}$ \\
\hline Fution \\
\hline
\end{tabular}

Fuente: elaboración propia.

\section{Visión global de la cultura tributaria}

El cumplimiento voluntario de las obligaciones tributarias y la toma de conciencia sobre la importancia de pagar impuestos, le permite al Estado obtener los recursos para satisfacer las necesidades públicas. Se requiere, además, de un proceso comunicacional que posibilite difundir toda la información relacionada con los tributos, y ejecutar estrategias educativas que permitan formar a los niños y jóvenes en esa cultura del deber ciudadano.

De acuerdo con un estudio sobre el panorama fiscal de la CEPAL (2018), varios países de la región mantuvieron un alto nivel de activismo tributario durante el año 2017. Muchos implementaron e identificaron reformas y medidas de política fiscal relacionadas con el pago de los impuestos. Igualmente, la cultura tributaria es vista como una política primordial en varios países, sin embargo, el objetivo principal difiere en cada uno de ellos, algunos buscan aumentar la base de contribuyentes, mientras que otros esperan aumentar el recaudo. Algunos países iberoamericanos utilizan estrategias dinámicas a través de programas de educación fiscal, dirigidas en su mayoría a la población juvenil, con el propósito de estimular la cultura tributaria en los futuros contribuyentes.

De igual manera, diferentes gobiernos a nivel mundial han establecido tácticas para implementar la cultura tributaria, tal es el caso de Sudáfrica que ha intensificado su presencia en zonas rurales, por medio de vehículos móviles que proporcionan información fiscal. Igualmente, en Zambia se instauró un programa radiofónico que les permite a los contribuyentes estar en contacto con la información fiscal y aclarar dudas respecto a estos temas. En Costa Rica se ha abierto un espacio lúdico, donde se educa a la población infantil mediante juegos de temática fiscal, con la finalidad de resaltar la importancia que tienen los impuestos para el país (Riveros, 2017).

Conforme a las Estadísticas tributarias en América Latina y el Caribe 2018, el promedio de ingresos por impuestos en Latinoamérica es 22,7\%, y el de la Organización para la Cooperación y el Desarrollo Económico (OCDE) de 34,3\%; es así que el panorama en América Latina muestra la necesidad de cambios, pues "la recaudación promedio fue de 1,8\% del PIB, lejos del 8,4\% de países de la OCDE” (El Tiempo, 2018, on line), 
siendo en Latinoamérica donde se registra un incumplimiento de las obligaciones tributarias, relacionado con la crisis de los valores democráticos y con la falta de solidaridad de algunos sectores de la población, los cuales son percibidos como motivos por los que la población no paga impuestos. Estos factores pueden ayudar a explicar la alta tolerancia de la evasión fiscal que se registra en América Latina (Díaz \& Lindemberg, 2014).

Existe la voluntad... expresada por la mayoría de los hogares, de pagar más impuestos en la medida en que mejore la calidad de los servicios públicos de salud, educación y seguridad, y haya menos corrupción y más control de la evasión. (CEPAL, 2018)

Por otra parte, en Colombia la Dirección de Impuestos y Aduanas Nacionales (DIAN), creó la firma digital para facilitar a los contribuyentes la información correspondiente al cumplimiento de las obligaciones tributarias, con el propósito de simplificar los procedimientos, generando un mayor índice de cumplimiento voluntario y fiscal.

En Bogotá, el proyecto de cultura tributaria tuvo sus inicios en el marco de la llamada Cultura Ciudadana. Los gobiernos posteriores mantuvieron dicho concepto acudiendo a diferentes estrategias. Durante el gobierno de Antanas Mockus (2001-2003) se buscó luchar contra la evasión e incentivar el pago oportuno. Posteriormente, en el programa de gobierno de Luis Eduardo Garzón (2004-2007), se intentó fortalecer la cultura tributaria a través de estrategias educativas dirigidas a todos los ciudadanos (Camacho \& Patarroyo, 2017).

Dentro de los estudios enfocados en el análisis de la cultura tributaria a nivel internacional se encuentra el trabajo desarrollado por Burga (2015), en el cual se concluye que los contribuyentes no cumplen con las obligaciones tributarias debido a una inadecuada gestión de los recursos por parte del Estado, por lo que enfatizan en la importancia de fomentar conocimientos de cultura tributaria a través de actividades lúdicas que motiven a los comerciantes al cumplimiento adecuado de sus obligaciones.

A nivel nacional, Camacho \& Patarroyo (2017), indican que la cultura tributaria es una herramienta fundamental que le permite al Estado generar un desarrollo económico, social, cultural y político, según la investigación enfocada a analizar el perfil del contribuyente en relación con la cultura tributaria en Colombia, mediante la identificación de las estrategias y acciones frente a la misma y la determinación de los factores que influyen en el comerciante.

A nivel local, Ortiz (2016) identificó las causas del incumplimiento del pago del impuesto de industria y comercio, en las que menciona la evasión del mismo, falta de compromiso tributario y carencia de sensibilización por parte de la Alcaldía Municipal frente a la importancia del pago del tributo en el municipio de Ocaña.

\section{La obligación tributaria en Ocaña}

En Colombia, existen impuestos departamentales y municipales los cuales se encargan de gravar la realización de determinadas actividades o la propiedad de ciertos bienes a fin de obtener recursos financieros para hacer frente a los gastos públicos y promover el desarrollo local y departamental (Solís, 2013). Con relación a Ocaña, los impuestos municipales se encuentran estipulados en el Acuerdo No. 42 de 2009 donde se adopta el Estatuto de Rentas del municipio.

Por tal motivo, es concerniente tener presente el concepto de los impuestos de industria y comercio, su complementario avisos y tableros, y el predial unificado, pues representan la base para el desarrollo de la investigación.

El impuesto de industria y comercio es aquel que se genera por el ejercicio o realización directa o indirecta de cualquier actividad industrial, comercial o de servicios en un determinado municipio y se causa así la persona posea o no establecimiento de comercio. Parafraseando a la Cámara de Comercio de Bogotá (2019), lo recaudado por este impuesto se destina, entre otros rubros, al pago de servicios públicos y a atender las necesidades de la comunidad, realizando la destinación de estos recursos a lo establecido en el plan de 
desarrollo municipal, que se distribuye presupuestalmente en el plan de inversiones. Teniendo en cuenta el hecho generador del impuesto de industria y comercio se ejecutó un análisis de la cultura tributaria de las personas que ejercen actividades comerciales, pues se encuentran obligadas por la ley a cumplir con el respectivo pago del impuesto. Igualmente, es pertinente considerar las actividades económicas con el fin de categorizarlas para describir los aspectos relacionados con su objeto social.

Complementario a este impuesto se encuentra el impuesto de avisos y tableros, el cual se paga por la instalación de avisos como vallas, tableros y emblemas, ubicados en la vía pública, en lugares públicos o privados, o en cualquier clase de vehículo visibles desde el espacio público. Este se liquida a la tarifa del $15 \%$ tomando como base el impuesto a cargo total del impuesto de industria y comercio (ICA) (Concejo Municipal de Ocaña, 2018).

Por su parte, el impuesto predial unificado es un tributo de carácter municipal que grava la propiedad inmueble, tanto urbana como rural que cobra el municipio sobre el avalúo catastral o sobre el valor de la declaración privada del mismo (Concejo Municipal de Ocaña, 2009). Al considerar el impuesto predial como fuente de recursos del municipio, la investigación analizó el conocimiento por parte de los comerciantes, el nivel de pago y los motivos por los cuales no realizan un efectivo recaudo, esto con el fin de conocer el nivel de cultura tributaria, siendo conscientes que el Estado es el encargado de generar procesos e instrumentos para el incremento de esta cultura y para la prevención de los delitos fiscales (Viktorovich, Mikhaylovich, Ziyavutdinovich et al., 2018).

\section{Metodología}

Esta investigación se formuló desde un nivel descriptivo para analizar la cultura tributaria de los comerciantes con respecto al pago de los impuestos de industria y comercio, avisos y tableros y el predial unificado en una zona de conflicto. Sabiendo que "con los estudios descriptivos se busca especificar las propiedades, las características y los perfiles de personas, grupos, comunidades, procesos, objetos o cualquier otro fenómeno que se someta a un análisis" (Sampieri, Fernández, \& Baptista, 2014, p. 92).

Se llevó a cabo un trabajo de campo para recolectar la información directamente de los comerciantes ubicados en la zona centro de Ocaña, responsables del pago de los impuestos de industria y comercio, avisos y tableros y predial unificado (Arias, 2012). Se siguió un enfoque cuantitativo orientado a analizar la cultura tributaria de los comerciantes, midiendo las variables de forma objetiva y con un alto grado de precisión, posteriormente se unificaron los resultados obtenidos mediante tablas y figuras para el respectivo análisis.

Una vez determinada la metodología a utilizar y de acuerdo con los objetivos planteados, se estableció la muestra con relación a la población estudiada según el planteamiento del problema, a saber, los comerciantes de la zona centro de la ciudad de Ocaña representados en 374, según información de la Cámara de Comercio de Ocaña (2018), constituyendo esta la población a estudiar.

Se estableció un tipo de muestreo probabilístico utilizando métodos estadísticos que arrojaron como resultado una muestra de 190 contribuyentes de la zona centro, a la cual se le aplicó una encuesta que constituyó el medio de fuente primaria para el análisis del nivel de cultura tributaria que poseen los comerciantes de esta zona. Una vez recopilada la información cualitativamente de las fuentes primarias se definieron los criterios para ordenar los datos obtenidos del trabajo de campo y posteriormente se analizaron los datos cuantitativamente a través de tablas y figuras, por medio de Microsoft Excel.

\section{Resultados y discusión}

Con miras a conocer la población estudiada y describir sus características se procedió a categorizar las actividades económicas que desempeñan las empresas de la zona centro de la ciudad de Ocaña, obteniendo 
como resultado que las más frecuentes son las correspondientes a las prendas de vestir CIIU 4771, piñaterías-misceláneas CIIU 4769-4719, droguerías-perfumerías CIIU 4773, y calzado CIIU 4772, las cuales representan el 59\% de las actividades que se realizan en el tejido empresarial estudiado, correspondiente al $28 \%$ prendas de vestir, $13 \%$ piñaterías y misceláneas, $11 \%$ droguerías y perfumerías y el $7 \%$ calzado, respectivamente. Por tanto, las empresas que ejercen estas actividades tienen gran importancia en el comercio de Ocaña, y están sujetas a una de las tarifas del impuesto de industria y comercio, avisos y tableros más altas aplicadas al sector comercial en la ciudad. La información se puede apreciar en la tabla 3.

TABLA 3

Tarifa del impuesto de Industria y Comercio para la actividad comercial

\begin{tabular}{clc}
\hline Código & \multicolumn{1}{c}{ Actividad comercial } & Tarifa \\
\hline 201 & $\begin{array}{l}\text { Venta de alimentos y productos agrícolas en bruto, } \\
\text { venta de textos escolares y libros }\end{array}$ & $3 \times$ mil \\
\hline 202 & $\begin{array}{l}\text { Venta de madera y materiales para la construcción, } \\
\text { venta de automotores incluida las motocicletas }\end{array}$ & $5 \times$ mil \\
\hline 203 & $\begin{array}{l}\text { Venta de cigarrillos y licores, venta de } \\
\text { combustibles y derivados de petróleo, venta de } \\
\text { joyas, y venta de drogas y medicamentos, } \\
\text { compraventas o casas comerciales }\end{array}$ & $8 \times$ mil \\
\hline 204 & Demás actividades comerciales & $5 \times$ mil \\
\hline
\end{tabular}

Fuente: Concejo Municipal de Ocaña (2009).

Para establecer el nivel de cumplimiento de las obligaciones tributarias, en primer lugar, se tuvo en cuenta el grado de conocimiento que poseen los comerciantes en cuanto a los impuestos estudiados, de acuerdo con los regímenes a los que pertenecen. Es necesario resaltar que la ley de financiamiento aprobada el 19 de diciembre de 2018 en el Congreso introdujo cambios en el impuesto sobre las ventas. Entre ellos, la adición del parágrafo 3 al artículo 437 del Estatuto Tributario, en el cual se menciona la nueva expresión: responsables del IVA, unificando así los términos régimen simplificado y régimen común. Por consiguiente, en el presente artículo se utilizaron los términos responsables y no responsables para identificar al régimen común y al simplificado, respectivamente.

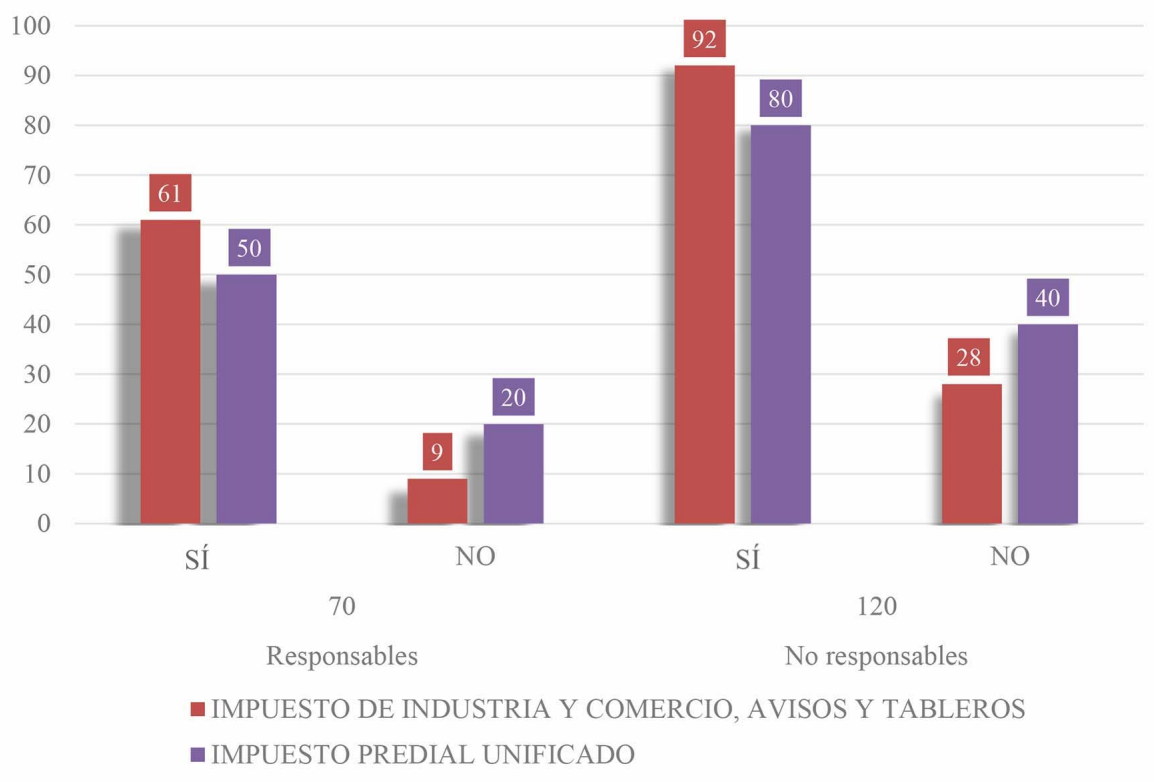

FIGURA 1

Grado de conocimiento de los comerciantes frente a los impuestos Fuente: elaboración propia con base en las encuestas aplicadas 
Como se aprecia en la figura 1, el régimen no responsable conoce menos acerca de los impuestos de industria y comercio, avisos y tableros y predial unificado, frente al régimen responsable. Sin embargo, la mayoría de los comerciantes tienen un conocimiento claro de los tributos, pues, en cuanto al impuesto de industria y comercio y su complementario avisos y tableros, del total encuestados, 153 tienen bien definido el concepto, y respecto al impuesto predial unificado 130 comerciantes comprenden su significado. Esto permite analizar que los contribuyentes de la zona centro están relacionados con los temas tributarios, por lo que están al tanto del tratamiento de estos impuestos.

TABLA 4

Comerciantes conscientes del pago de los impuestos

\begin{tabular}{crc}
\hline Rubro & Frecuencia & Porcentaje \\
\hline Sí & 187 & 98,42 \\
\hline No & 3 & 1,58 \\
\hline Total & $\mathbf{1 9 0}$ & $\mathbf{1 0 0 , 0 0}$ \\
\hline
\end{tabular}

Fuente: elaboración propia con base en las encuestas aplicadas.

En segundo lugar, se determinó la aceptación que tienen los comerciantes de cumplir las obligaciones tributarias con el fisco, demostrando que el 98,42\% de los encuestados son conscientes del deber que tienen de pagar los impuestos y tan solo el 1,58\% no lo son. "Se busca, entonces, que la normatividad sea asimilada por los individuos y expresada en sus acciones. El contribuyente que acepta voluntariamente el pago lo hace por sí mismo y por la sociedad, más allá de si existe una sanción” (Bonilla, 2014, p. 23). Por esa razón, se puede determinar que la mayoría de los comerciantes asimila las responsabilidades que tienen para con el Estado al ejercer una actividad económica en la jurisdicción municipal.

Luego, para determinar el grado de responsabilidad de las personas que ejercen una actividad de comercio en la zona centro de Ocaña, se analizó el compromiso que tienen frente al pago de los tributos municipales.

TABLA 5

Compromiso frente al pago del impuesto de industria y comercio

\begin{tabular}{ccc}
\hline Rubro & Frecuencia & Porcentaje \\
\hline Sí & 189 & 99,50 \\
\hline No & 1 & 0,50 \\
\hline Total & $\mathbf{1 9 0}$ & $\mathbf{1 0 0 , 0 0}$ \\
\hline
\end{tabular}

Fuente: elaboración propia con base en las encuestas aplicadas.

Primero, en cuanto al impuesto de industria y comercio, avisos y tableros, se identifica, que tan solo un comerciante no realiza el pago de este tributo, por lo que el 99,5 \%sí lo realizan, demostrando así que los comerciantes están comprometidos en dar cumplimiento a esta obligación tributaria.

TABLA 6

Compromiso frente al pago del impuesto predial unificado

\begin{tabular}{ccc}
\hline Rubro & Frecuencia & Porcentaje \\
\hline Sí & 33 & 17,40 \\
\hline No & 157 & 82,60 \\
\hline Total & $\mathbf{1 9 0}$ & $\mathbf{1 0 0 , 0 0}$ \\
\hline \multirow{2}{*}{ Fuente: elaboración propia con base en las encuestas aplicadas. }
\end{tabular}

Respecto al compromiso que tienen los comerciantes frente al pago del impuesto predial, se percibe que el $82,6 \%$ no realiza el pago del impuesto, debido a que no son propietarios del inmueble donde ejercen la actividad económica. 


\section{Nivel de cumplimiento de las obligaciones tributarias}

Los criterios para determinar el nivel de cumplimiento de las obligaciones tributarias se establecen con base en la clasificación de los componentes de la cultura tributaria, donde es necesario que haya una presentación oportuna de las declaraciones de impuestos, su pago oportuno y la presentación en la unidad tributaria (Niknamian, 2017). Por consiguiente, el nivel bajo se conforma con pagar la obligación, para el nivel medio se requiere tener un compromiso y asimilar la obligación, es decir, ser consciente del deber de tributar y para tener un nivel alto se demanda tener un conocimiento general de los impuestos, aceptar y ejecutar el pago de los mismos. La información se puede apreciar en la tabla 7.

TABLA 7

Criterios para la determinación del nivel de cumplimiento

\begin{tabular}{lccc}
\hline \multicolumn{1}{c}{ Rubro } & Alto & Medio & Bajo \\
\hline Conocimiento & $\mathrm{X}$ & & \\
\hline Aceptación & $\mathrm{X}$ & $\mathrm{X}$ & \\
\hline Compromiso & $\mathrm{X}$ & $\mathrm{X}$ & $\mathrm{X}$ \\
\hline
\end{tabular}

Fuente: elaboración propia con base en las encuestas aplicadas.

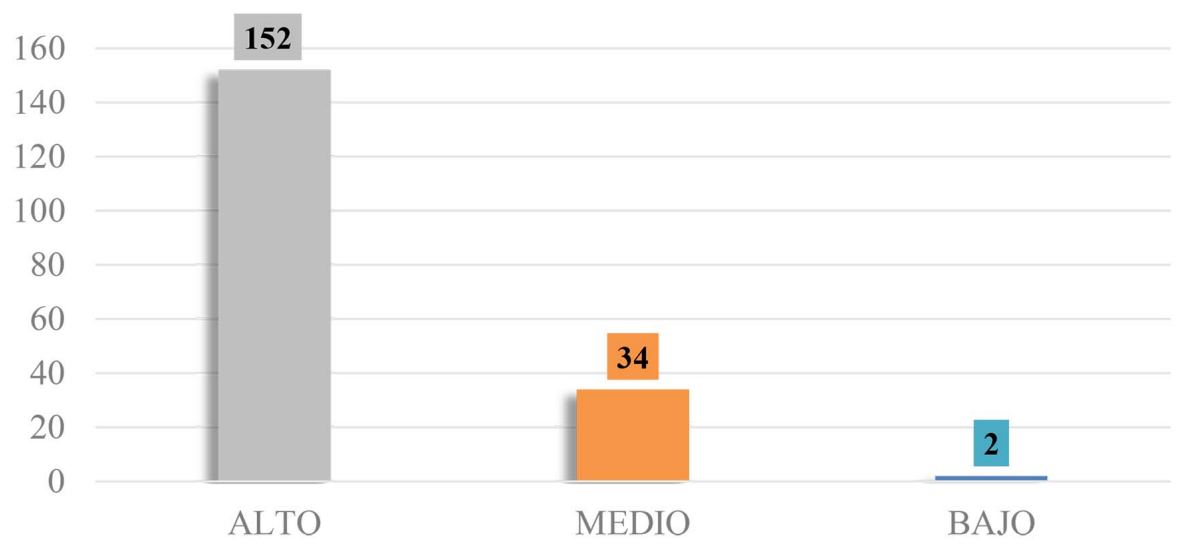

FIGURA 2

Nivel de cumplimiento de los comerciantes frente al impuesto de industria y comercio Fuente: elaboración propia con base en las encuestas aplicadas.

En la figura 2 se observa que 152 comerciantes tienen un nivel de cumplimiento alto, 34 aceptan y cumplen con el pago de los impuestos y dos tienen un nivel bajo, es decir, solo realizan el pago del impuesto sin tener conocimiento de ellos ni ser conscientes del deber que tienen al ejercer una actividad económica. Se especifica que de los 190 comerciantes encuestados, dos no cumplen con ninguno de los criterios establecidos debido a que uno solamente es consciente, es decir, acepta el pago del impuesto, y el otro conoce y cumple mas no es consciente del deber de tributar. De esta manera se determina que el 80,9\% de los comerciantes de la zona centro, al tener un nivel de cumplimiento alto, demuestran un compromiso en los temas tributarios. 


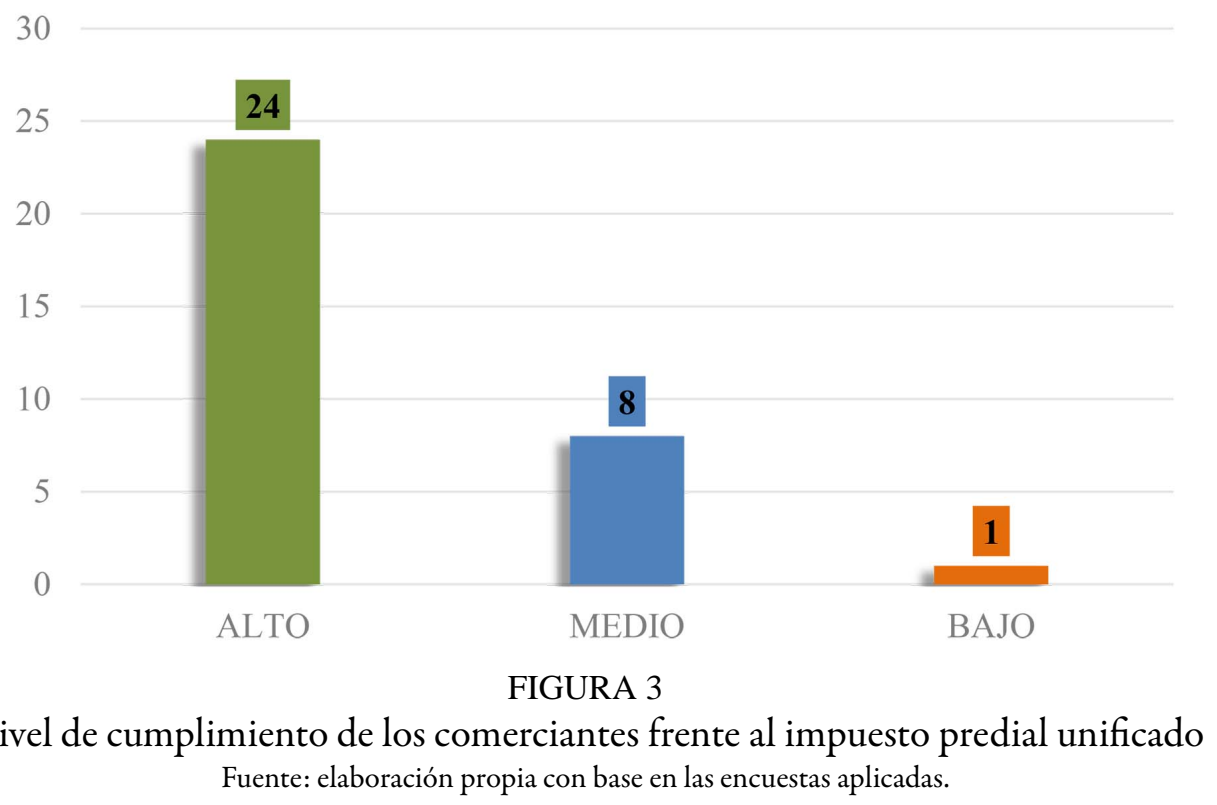

Se puede apreciar en la figura 3, que un comerciante encuestado tiene un nivel de cumplimiento bajo, ocho están en la categoría media y 24 tienen un nivel de cumplimiento alto, es decir, que conocen el significado de los impuestos analizados, asimilan que son responsables de tributar y cumplen con el pago de estas obligaciones. Es necesario resaltar que 33 comerciantes tienen un nivel de cumplimiento, puesto que son propietarios del inmueble y por ende son responsables del pago del impuesto predial.

\section{Causas del cumplimiento o evasión de los impuestos}

Con el propósito de identificar los motivos por los cuales los comerciantes cumplen o evaden las responsabilidades tributarias con la ciudad, fue pertinente conocer la argumentación que tienen frente al pago y evasión de los impuestos. Ver la figura 4

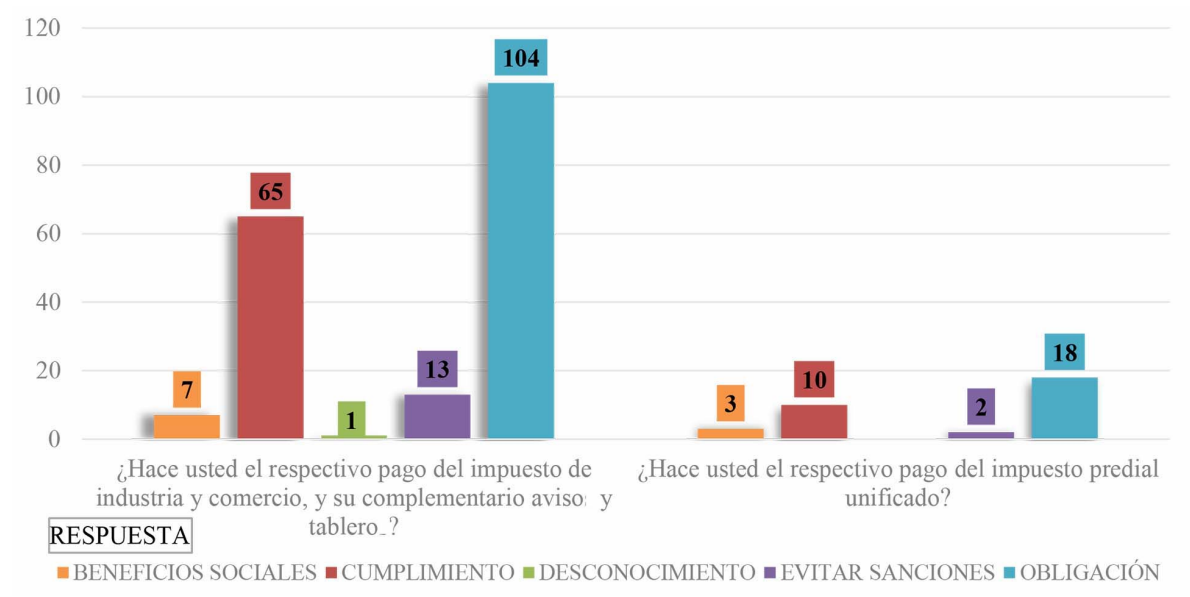

FIGURA 4

Causas del cumplimiento o evasión de los impuestos Fuente: elaboración propia con base en las encuestas aplicadas.

Se puede percibir que tan solo un comerciante no realiza el respectivo pago del impuesto de industria y comercio, debido al desconocimiento que posee en cuanto a sus obligaciones, demostrando que es esencial 
apropiarse de los temas tributarios al momento de comenzar un emprendimiento, para no llegar a tener algún inconveniente económico, pues "el desconocimiento de la ley fiscal no exime de la obligación de cumplir con ella, y de las sanciones monetarias ocasionadas por no hacerlo” (Hermida, 2018, p. 1).

En cuanto a las causales de cumplimiento se aprecia que la mayoría de los comerciantes pagan los impuestos por obligación; no de manera voluntaria, pues a pesar de que el impuesto es una imposición legal, por lo que representan una obligación, en las sociedades democráticas los ciudadanos deben ser conscientes de sus derechos y obligaciones. La finalidad de la cultura tributaria es que el impuesto como obligación se transforme en un autoimpuesto asumido por los contribuyentes como un compromiso social. "Tradicionalmente se ha creído que el factor determinante del cumplimiento es la capacidad coercitiva de la autoridad fiscal, traducida en el temor del ciudadano a ser sancionado sino cumple con su obligación (llamada percepción de riesgo)" (Estrada, 2014, p. 14).

Además se identificó que 13 comerciantes en relación con el impuesto de industria y comercio y tan solo dos en cuanto al impuesto predial pagan para evitar sanciones, las cuales podrían perjudicar las actividades diarias de la empresa o incluso podría llevar al cierre temporal o definitivo del establecimiento. Así, estos comerciantes no efectúan el pago de los tributos de manera voluntaria, lo que demuestra una falta de apropiación de la importancia de los impuestos, pues no lo perciben como un beneficio social.

Por el contrario, 72 comerciantes realizan el pago del impuesto de industria y comercio y 13 respecto al impuesto predial unificado, demostrando sensatez en cuanto al deber que tienen de aportar económicamente los recursos que la ciudad necesita para invertir en obras que beneficien a la comunidad. Sin embargo, tan solo el 20\% de este recaudo es invertido en pavimentación de vías, sector agropecuario, equipamiento municipal, acueducto y alcantarillado y grupos vulnerables (Bayona, 2019); los demás recursos son destinados al gasto de funcionamiento de Alcaldía, es decir, el pago de nómina y honorarios de los funcionarios públicos tanto de la administración central como de los dos órganos de control, a saber, el concejo y la personería municipal.

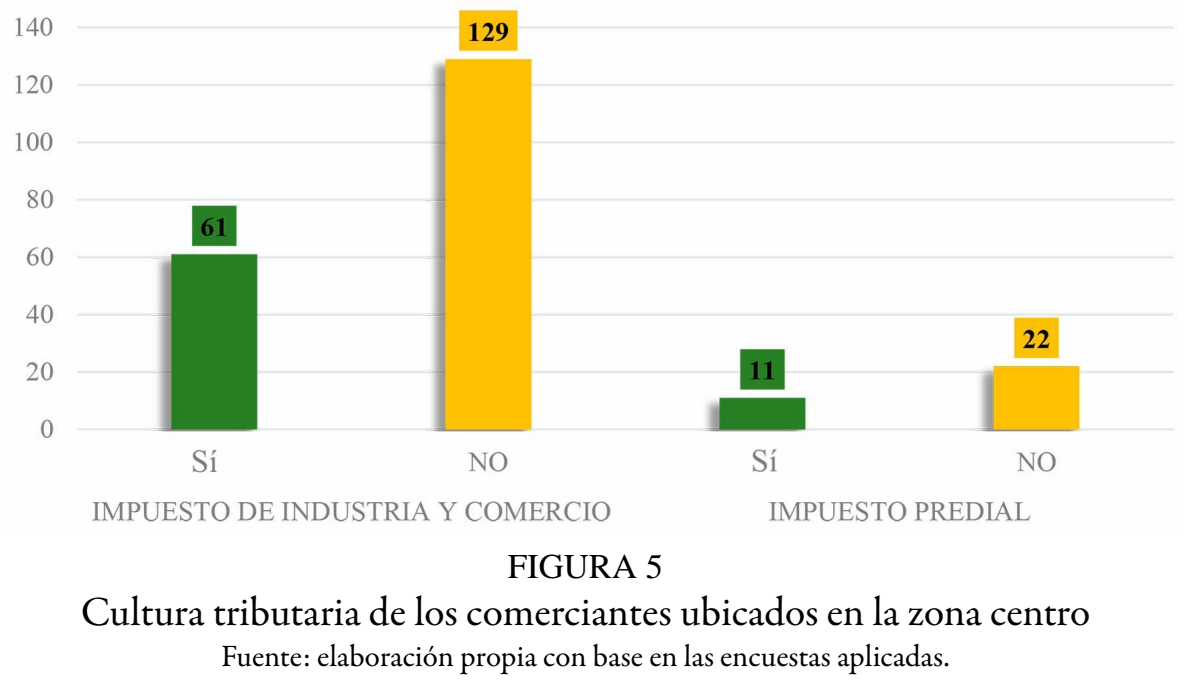

En la figura 5 se puede observar que 61 comerciantes tienen cultura tributaria en cuanto al impuesto de industria y comercio, y más del 50\% de los comerciantes no realizan el pago de manera voluntaria y consciente. Igualmente, frente al impuesto predial, del total de comerciantes responsables del tributo tan solo 11 tienen cultura tributaria, pues su nivel de cumplimiento es alto y no realizan el pago por obligación ni para evitar sanciones.

Finalmente, estos mercaderes no representan un porcentaje tan alto en la zona centro, frente a aquellos que no poseen cultura tributaria. Se infiere que muy pocos confían en que los recursos recaudados serán utilizados de manera adecuada en proyectos que retribuyan positivamente en el desarrollo de la región. 
Es necesaria la aplicación equitativa de políticas públicas locales de desarrollo, articuladas con instituciones del nivel nacional y departamental, de tal manera que se generen espacios de participación ciudadana para identificar y priorizar estrategias que conduzcan al municipio hacia el desarrollo social y económico sustentable. (Cañizares et al., 2015, p. 120)

\section{Conclusiones}

Para estimar la cultura tributaria de los comerciantes del centro de Ocaña se hizo una revisión de las empresas ubicadas en la zona objeto de estudio y se identificó que más del $50 \%$ de las empresas se dedican al comercio de prendas de vestir, calzado, medicamentos farmacéuticos y artículos decorativos. Es fundamental que estas empresas posean cultura tributaria para que exista mayor apropiación en cuanto al cumplimiento voluntario de sus responsabilidades.

Para la determinación del nivel de cumplimiento de los comerciantes se tuvo en cuenta el conocimiento, la aceptación y el compromiso en cuanto a los impuestos analizados, de lo cual se pudo concluir que el $80,9 \%$ y el $72,7 \%$ de los contribuyentes tienen un nivel de cumplimiento alto en relación con el impuesto de industria y comercio y el predial unificado, respectivamente, pues obedecen con los criterios establecidos para su medición. Esto traduce que un alto número de comerciantes de la zona centro de Ocaña conocen los conceptos relacionados con los impuestos, son conscientes del deber que tienen con la ciudad al ejercer una actividad económica y realizan los pagos establecidos en las fechas asignadas, demostrando un alto grado de responsabilidad.

En cuanto a las razones por las cuales los contribuyentes realizan el pago de los impuestos, se identificó que el 37,9\% en cuanto al impuesto de industria y comercio y el 39,4\% con respecto al impuesto predial unificado, lo ejecutan para cumplir con la tributación y para obtener beneficios sociales, lo que manifiesta la relevancia que tiene para los comerciantes realizar el pago de la gabelas municipales para el sostenimiento y desarrollo de la región.

Por consiguiente, teniendo en cuenta las causas del pago y el nivel de cumplimiento, se analizó la cultura tributaria de los comerciantes, de lo cual se concluyó que el 32,1\% tiene cultura tributaria frente al impuesto de industria y comercio y el 33,3\% referente al predial unificado. Por tanto, la cultura tributaria de los comerciantes ubicados en la zona centro de Ocaña es baja pues menos del 50\% realizan el pago de manera voluntaria. Por tanto, es necesario que el Estado garantice el mejoramiento de las condiciones de vida, de tal manera que se fortalezca el desempeño del sector productivo y se vea reflejado en un aumento del compromiso en el cumplimiento del pago voluntario de los impuestos de industria y comercio, avisos y tableros y del predial unificado.

\section{Consideraciones éticas}

El desarrollo del proceso de investigación que dio resultado al artículo utilizó fuentes de información primarias con la aplicación de encuestas a los comerciantes ubicados en el centro de la ciudad de Ocaña, Norte de Santander, realizando las fases del proyecto con valores éticos frente a la recolección de la información, a la presentación de los resultados, reflejando un trato digno, con respeto, sin vulnerar los derechos éticos de las organizaciones encuestadas.

\section{Contribución de los autores}

Los autores del artículo participaron por partes iguales en el proyecto de investigación, desde la fase del diseño de la propuesta, el diseño metodológico, la presentación de resultados y la elaboración del manuscrito. 


\section{Financiación}

Para el desarrollo del proceso de investigación los recursos utilizados fueron generados con aporte en especie, horas de dedicación de investigación de la docente Naydu Judith Jácome docente de planta de la Universidad Francisco de Paula Santander Ocaña y los investigadores Yasneidy Lorena Peñaranda e Ilver Jaimes.

\section{Conflictos de interés}

Los autores declaramos que ninguno tiene relación o conflicto de interés asociado con el proceso de la investigación ni con la presentación del artículo.

\section{Referencias}

Abondano, A., Peña, R. d., \& Parada, M. (2018, 21 de febrero). La importancia del impuesto predial para la igualdad en Colombia. El Espectador, p. 1. https://www.elespectador.com/colombia20/analistas/la-importancia-del-im puesto-predial-para-la-igualdad-en-colombia-article.

Acosta, G., Ramírez, R., \& Maldonado, E. (2017). Niveles de cumplimiento tributario relacionado con el impuesto al valor agregado. Revista Polo del Conocimiento, 2(6). https://doi.org/10.23857/pc.v2i6

Actualícese (2017, 14 de marzo). Actualicese. https://actualicese.com/obligacion-formal-y-sustancial-conceptos/

Arias, F. (2012). El proyecto de investigación, 6a edición. Caracas: Episteme.

Bayona, W. (2019, 22 de mayo). Destinación de los recursos de los impuestos de industria y comercio y predial unificado. Entrevista de Y. L. Rizo, \& I. Jaimes. Ocaña, Norte de Santander.

Bonilla, E. (2014). La cultura tributaria como herramienta de política fiscal, con énfasis en la experiencia de Bogotá. Revista Ciudad. Estados Politica, pp. 1-23. https://revistas.unal.edu.co/index.php/revcep/article/view/44456

Burga, M. (2015). Cultura tributaria y obligaciones tributarias en las empresas comerciales del emporio Gamarra 2014. Lima: Universidad de San Martín de Porres.

Camacho, A., \& Patarroyo, Y. (2017). Cultura tributaria en Colombia. Bogotá: Universidad Minuto de Dios.

Cámara de Comercio de Bogotá (2019, 7 de febrero). Cámara de Comercio de Bogotá. https://www.ccb.org.co/Pregu ntas-frecuentes/Tramites-registrales/En-que-consiste-el-impuesto-de-industria-y-comercio

Cámara de Comercio de Ocaña (2018, 9 de noviembre). Comerciantes ubicados en la zona centro del municipio de Ocaña (Base de Datos). Ocaña: Cámara de Comercio.

Cañizares, J. d., Cárdenas, M., \& Velasco, B. (2015, 29 de octubre). Impacto de la violencia política en la gobernabilidad del municipio de Ocaña (Colombia). Recuperado el 27 de agosto de 2019 de http://www.scielo.org.co/pdf/peg e/n39/n39a07.pdf

Centro Nacional de Memoria Histórica (2017). Centro de Memoria Histórica. Recuperado el 27 de agosto de 2019 de http://centrodememoriahistorica.gov.co/micrositios/catatumbo/

Colmenero, R. (2015). Concienciación cívico-tributaria en el Siglo XXI. Anuario Jurídico y Económico Escurialense, 184.

Comisión Económica para América Latina y el Caribe - CEPAL (2018). Panorama Fiscal. Los desafios de las políticas públicas en el marco de la Agenda 2030. https://repositorio.cepal.org/bitstream/handle/11362/43405/7/S180 0082_es.pdf

Concejo Municipal de Ocaña (2009, diciembre). Estatuto Tributario del Municipio de Ocaña, Norte de Santander. Acuerdo 042 de 2009. Ocaña: Alcaldía Municipal. https://notinet.com.co/verdes_impuesto.php?taxesdep=52 10

Concejo Municipal de Ocaña (2018). Acuerdo 002 del 18 de diciembre de 2018. Ocaña: Administración Municipal. 
Concha, T., Ramírez, J., \& Acosta, O. (2017). Tributación en Colombia: reformas, evasión y equidad. Santiago: CEPAL. https://repositorio.cepal.org/bitstream/handle/11362/43133/1/S1700948_es.pdf

Congreso de la República de Colombia (1990, 18 de diciembre). Ley 44 de 1990, Por la cual se dictan normas sobre catastro e impuestos sobre la propiedad raíz, se dictan otras disposiciones de carácter tributario, y se conceden unas facultades extraordinarias. Bogotá: Congreso de la República de Colombia.

Constitución Política de Colombia (1991). Constitución Politica de Colombia. República de Colombia. Bogotá: Atenea Ltda.

Departamento Administrativo de Hacienda y Secretaría de Educación Municipal de Cali. (2015, 29 de abril). Cartilla Cultura Tributaria. Cali: Lito Muñoz S.A.S.

Departamento Nacional de Planeación - DNP (2015). Desempeño fiscal de los departamentos y municipios. Bogotá: DNP. https://colaboracion.dnp.gov.co/CDT/Desarrollo\%20Territorial/Desempe\%C3\%B1o\%20Fiscal\%202 015\%20V.2016.11.09.pdf

Díaz, F. d., \& Lindemberg, A. (2014, 1 de noviembre). Educación fiscal. Recuperado el 15 de febrero de 2019 de http: //www.educacionfiscal.org/files/2016-08/cultura-contributiva-en-america-latina-livro-mexico.pdf

Dirección de Impuestos y Aduanas Nacionales - DIAN (2020). Estatuto Tributario. Bogotá: Legis.

Economipedia (2016, 6 de diciembre). Haciendo fácil la Economía. Recuperado el 21 de septiembre de 2018 de http: //economipedia.com/definiciones/obligacion-tributaria.html

El Tiempo (2018, 27 de marzo). En 2016, IVA fue la mayor fuente de ingresos tributarios en la región. El Tiempo. https://www.eltiempo.com/economia/sectores/estadisticas-tributarias-en-america-latina-y-el-carib e-2018-por-la-ocde-198886

Estrada, S. (2014). Cultura tributaria: 50 preguntas y respuestas. Guatemala: Superintendencia de Administración Tributaria.

Gerencie (2020, 16 de agosto). Obligaciones tributarias formales Gerencie. https://www.gerencie.com/obligaciones-tr ibutarias-formales.html\#: :text=Obligaci\%C3\%B3n\%20tributaria\%20formal.\&text=Una\%20obligaci\%C3\% B3n\%20formal\%20es\%20aquelpara\%20cumplir\%20con\%20determinadas\%20obligaciones

Hermida, C. (2018, 6 de febrero). Desconocer obligaciones fiscales no exime de su cumplimiento. Universo. https://w ww.uv.mx/prensa/general/desconocer-obligaciones-fiscales-no-exime-de-su-cumplimiento/

La Opinión (2018, 19 de enero). 5 mil millones en cartera morosa en Ocaña. La Opinión, p. 1.

Niknamian, S. (2017). Investigating tax culture of the tax payers of the Iranian Tax Administration. Washington D.C.: SSRN.

Ortiz, L. (2016). Análisis de la cultura tributaria de los contribuyentes en el mercado público del municipio de Ocaña frente al impuesto de industria y comercio. Ocaña: Universidad Francisco De Paula Santander Ocaña.

Páez, L. (2014, 22 de junio). Academia de Historia de Ocaña. Recuperado el 14 de septiembre de 2018 de https://aca demiaocana.blogspot.com/2014/07/v-behaviorurldefaultvmlo.html

Portafolio-Impuestos (2019, 26 de marzo). Colombia sigue atrás en el cobro de los impuestos. Portafolio. https://ww w.portafolio.co/economia/impuestos/colombia-sigue-atras-en-el-cobro-de-los-impuestos-527866

Ramos, A., \& Remigio, Y. (2015). La cultura tributaria como estrategia para disminuir la evasión fiscal en las empresas comerciales de la provincia de Huaura. Huacho: Universidad Nacional José Faustino Sánchez Carrión.

Riveros, M. (2017). Como se debe implementar la cultura tributaria en Colombia. Bogotá: Universidad Militar Nueva Granada.

Sampieri, R., Fernández, C., \& Baptista, P. (2014). Metodología de la investigación, 6ª ed. México D.F.: Interamericana Editores, S.A. y McGraw-Hill.

Secretaría de Hacienda Distrital (2008). Industria y Comercio, avisos y tableros, ICA. http://www.shd.gov.co/shd/s ites/default/files/files/contabilidad/ica.pdf

Solís, M. (2013, 2 de febrero). Propuesta para el mejoramiento de la recaudación tributaria en materia de impuesto sobre las actividades económicas. Caracas: Universidad Católica Andrés Bello. 
Viktorovich, V., Mikhaylovich, D., Ziyavutdinovich, K., Agaverdievna, S., \& Petrovna, I. (2018). Tax culture in the system of countering informal business activities. Research Journal of Pharmaceutical, Biological and Chemical Sciences, 9, 1867-1871.

Villasmil, M., Fandiño, Y., \& Alvarado, L. (2018). Cultura tributaria en la educación: Un estudio fenomenológico hacia una interpretación compleja. Revista de Ciencias Humanas y Sociales, 34(18), 1620-1652. https://www.researchgate.net/publication/331907019_Cultura_tributaria_en_la_educacion_Un _estudio_fenomenologico_hacia_una_interpretacion_compleja

\section{Notas}

* Artículo de investigación

\section{Licencia Creative Commons CC BY 4.0}

Sugerencia de citación: Jácome, N., Rizo, Y., \& Jaimes, I. (2021). Análisis de la cultura tributaria de los comerciantes en zona de conflicto. Caso comerciantes ubicados en el centro de la ciudad de Ocaña, Norte de Santander. Cuadernos de Contabilidad, 22. https://doi.org/10.11144/Javeriana.cc22.actc 